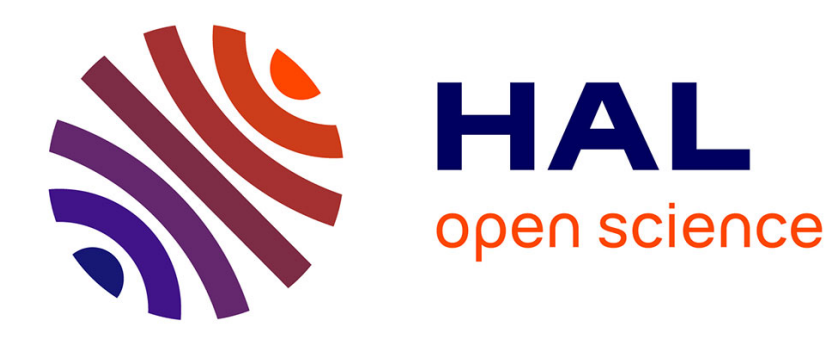

\title{
Management réformateur et utopie rationnelle
}

Jean-Luc Metzger

\section{To cite this version:}

Jean-Luc Metzger. Management réformateur et utopie rationnelle. Cahiers Internationaux de Sociologie, 2001, 111 (2), pp.233-259. halshs-00456291

\section{HAL Id: halshs-00456291 https://shs.hal.science/halshs-00456291}

Submitted on 17 Feb 2010

HAL is a multi-disciplinary open access archive for the deposit and dissemination of scientific research documents, whether they are published or not. The documents may come from teaching and research institutions in France or abroad, or from public or private research centers.
L'archive ouverte pluridisciplinaire HAL, est destinée au dépôt et à la diffusion de documents scientifiques de niveau recherche, publiés ou non, émanant des établissements d'enseignement et de recherche français ou étrangers, des laboratoires publics ou privés. 


\section{MANAGEMENT REFORMATEUR ET UTOPIE RATIONNELLE}

par Jean-Luc METZGER

RESUME

Cet article propose d'utiliser le concept d'utopie rationnelle pour rendre compte des pratiques managériales et tout particulièrement de la production de réformes répétées dans les organisations. Dans cette perspective, après avoir décrit une décennie de transformations au sein d'une organisation publique, certaines caractéristiques de la "culture managériale » sont dégagées de sa pratique réformatrice. Dans un second temps, le concept d'utopie rationnelle est construit à partir de textes canoniques et des travaux $d^{\prime}$ ' utopologues ». Dans un troisième temps, utopie rationnelle et culture managériale sont comparées, afin de préciser l'apport cognitif de cet outil à la compréhension du fonctionnement des organisations.

Mots clés : Management, Modernisation des entreprises, Organisations, Rationalité, Utopie.

\section{SUMMARY}

This article proposes to use the concept of utopia to account for the managerial practices and especially to explain the production of repeated organizational transformations. From this point of view, the case of a decade of ceaseless transformations into a public organization is first described. Some of the features of the "managerial culture" are then inferred from this empirical statement. In the second place, the concept of rational utopia is constructed, from canonical texts and works of "utopologists". Finally, rational utopia and managerial culture are compared, in order to specify the main cognitive contributions of this tool to the understanding of organizational functionning.

Key words : Management, Modernization of firms, Organizations, Rationality, Utopia.

On se propose, ici, de tester le caractère explicatif de la notion d'utopie dans le cadre de la pratique managériale. Pour ce faire, on voudrait vérifier que le «gouvernement» des entreprises, et plus précisément l'arrière-fond culturel à partir duquel il procède, est susceptible d'une interprétation en termes d'utopie. Cette réflexion s'inscrit dans l'ensemble des analyses portant sur la rationalité des décisions managériales et suggère ainsi d'échapper à l'alternative : ou bien la décision est considérée comme $a$ priori rationnelle (ne serait-ce que du point de vue de l'acteur) ; ou bien elle 
s'explique selon le «modèle de la poubelle» (la solution pré-existe au processus de décision, le processus lui-même semble obéir à une logique chaotique) $)^{1}$.

Dans cette perspective, nous allons tout d'abord décrire un cas empirique de transformation volontaire et incessante d'une organisation publique. A partir de ces éléments, nous identifierons les traits d'une culture managériale de la « réforme permanente ». Dans un deuxième temps, l'article présente les caractéristiques communes à certains récits utopiques, constituant ainsi un concept : celui de l'utopie rationnelle. En comparant ces deux éléments, nous chercherons ce que la pratique dirigeante a d'utopique et ce que le concept d'utopie peut apporter à l'analyse du changement volontaire.

\section{I. - CONTEXTE GENERAL \\ DE TRANSFORMATION DES ENTREPRISES PUBLIQUES}

La transformation des organisations publiques est, le plus souvent, présentée comme la solution à des dysfonctionnements structurels internes ou une réponse adaptative à l'environnement. Pourtant, l'environnement et ses évolutions sont des constructions sociales faisant suite, notamment, à des décisions prises au sein des grandes entreprises (Bauer et Bertin-Mourot, 1996, p. 68-70 ; Sulieman et Mendras, 1995, p. 47). De plus, «l'extérieur» constitue aussi bien une contrainte qu'une ressource (notamment, symbolique) pour les managers (Martin, 1998). C'est pourquoi, il nous semble utile, pour rendre compte de la pratique réformatrice, de prendre du recul par rapport aux discours managériaux et de considérer la volonté de transformer le social, comme un fait à expliquer et non comme d'emblée justifié.

L'organisation à la quelle nous nous référons a, pendant la décennie 1987-1997, connu de fortes évolutions que l'on peut classer en quatre catégories : a) changements de statut juridique ; b) changements de mode de gestion du personnel ; c) restructurations ; d) changements de l'organisation du travail. On notera que chaque catégorie est au pluriel, car ce qui est pertinent pour notre analyse, c'est le caractère permanent, récurrent, têtu de la volonté de conformer le social à un plan pré-établi ${ }^{2}$.

A / En effet, sur la période 1987-1995, cinq tentatives de privatisation se succédèrent, toutes précédées de "rapports" aux conclusions convergentes, mobilisant cinq ministres de tutelle, deux présidents de l'organisation et leurs états-majors, ainsi que d'innombrables "débats" internes, envisagés comme autant de projets à part entière. De leur côté, les salariés et les

\footnotetext{
${ }^{1}$ Pour une analyse de la décision selon le modèle de la poubelle voir MARCH et OLSEN, 1991.

${ }^{2}$ Pour une description plus détaillée de ces transformations, voir METZGER, 2000.
} 
organisations syndicales ont montré leur opposition à cet objectif, par plusieurs journées de grève nationales, fortement suivies (de 50 à $75 \%$ de participation) ${ }^{1}$. Néanmoins, un premier changement de statut eut lieu en 1991, faisant de l'administration une entreprise publique. Mais c'est à partir de 1996, après donc 9 ans de controverse, que la combativité fléchit ${ }^{2}$, en sorte que la dernière journée d'opposition à la privatisation ne mobilisa, en 1997, que $20 \%$ des salariés, alors que la vente d'actions en séduisit presque les trois quart.

B / Ce renversement est d'autant plus remarquable que, parallèlement, tous les aspects de l'activité professionnelle ont été modifiés. En effet, plusieurs autres réformes, concernant la gestion du personnel, furent mises en oeuvre, en introduisant : un mode d'affectation et de promotion réputés privilégier la reconnaissance des compétences (la gestion par fonction veut remplacer la gestion par grade); un mode de recrutement privilégiant le recours à des statuts d'emploi plus précaires ${ }^{3}$; la pratique d'entretiens de progrès, avec fixation d'objectifs ; une rémunération plus individualisée ; une procédure de départ en préretraite (à partir de 55 ans). Sans oublier que chacun de ces axes de modifications subit lui-même plusieurs adaptations, tant dans ses principes que dans sa mise en œuvre.

$\mathrm{C} /$ Simultanément, s'est opéré un réaménagement permanent des structures : création puis suppression de centres de coût internes ; séparations successives des activités réputées stratégiques et opérationnelles ; modification des zones d'intervention des établissements opérationnels; fusions successives des établissements ainsi créés, avec réduction des lignes hiérarchiques ; diminution graduelle des effectifs de la direction générale et son découpage en branches ; externalisation d'activités jugées hors du " cœur de métier"; contractualisation des prestations réciproques internes. Il faut tenir compte, également, des restructurations décentralisées, entreprises à l'initiative d'un management local plus autonome et soucieux de traduire, à son échelle, les " priorités" de la direction. Ces restructurations s'ajoutant et se combinant aux restructurations globales. En sorte que, des services entiers (parfois des filières professionnelles) sont soumis à des "sur-réformes", conduisant certains salariés à changer tous les six mois de service.

D / Pour mettre en oeuvre ces réformes, il fallut procéder à des modifications de l'organisation du travail, notamment par l'introduction de nouvelles technologies et l'informatisation croissante de domaines jusque là peu automatisés (vente, dépannage, informatique et formation internes), sans oublier l'allongement des plages d'ouverture de certains services. Là-dessus

\footnotetext{
${ }^{1}$ Source : Le Bilan social de l'entreprise publique, de 1987 à 1997.

2 La participation aux grèves nationales varie alors de 30 à $45 \%$ et plus généralement, le nombre de jours de grève par agent passe de 0,86 en 1987 à 0,37 en 1997.

${ }^{3}$ La population d'agents hors statut a triplé entre 1987 et 1997.
} 
se greffent des procédures de qualification et certification de certaines activités, obligeant à des "remises à plat" des pratiques, en vue de leur rationalisation. Enfin, la forte et rapide modification du contenu des emplois, obligeant plus de 30000 salariés à changer d'activité, de lieu de travail, de collègues, d'opportunité et de pratique, s'est traduite par un recours instrumental à la formation, dont les modalités et les finalités ont plusieurs fois changé pendant la même période. Précisons que cet important brassage a renforcé l'importance, dans la pratique, des hiérarchiques, seuls aptes à valider les mobilités professionnelles.

Ajoutons une dimension pour ainsi dire transverse, commune aux quatre familles de réformes, et qui concerne la volonté d'inculquer aux salariés une culture marchande, culture basée sur l'idée que, dorénavant, toutes les activités doivent être " pilotées par le client", qu'il soit externe ou interne (chaque service, chaque collègue est, idéalement, un client). Telle est, rapidement esquissée, l'ampleur des injonctions que les salariés ont dû rendre "localement" compatibles ${ }^{1}$.

Vis-à-vis d'un tel phénomène, certaines analyses sociologiques soulignent le caractère contingent ou pour le moins inattendu des décisions. Toutefois, cette approche ne permet pas d'expliquer deux choses : d'une part, l'étrange ressemblance de certaines décisions prises dans des entreprises différentes, par des hommes différents, raisonnant a priori à partir de données différentes, dans des contextes et pour des objectifs différents (c'est le cas des entreprises publiques en France); d'autre part, la récurrence, constatée sur une longue période et pour une même entreprise, de décisions prises par des hommes différents, décisions qui, mises bout à bout, se présentent comme le lent développement d'une pensée têtue. En d'autres termes, plutôt que de mettre l'accent sur les tensions entre acteurs décidant, mais sans pour autant nier le poids de leur rationalité limitée, nous allons nous attacher à comprendre ce qui peut s'analyser comme la manifestation d'une culture commune (dans la perspective de C. W. Mills, 1956), propre au groupe d'individus concevant et décidant de mettre en œuvre la succession des réformes.

\footnotetext{
${ }^{1}$ On notera que, si cette série de transformations volontaires a été décrite à propos d'une organisation publique donnée, elle n'a rien de proprement exceptionnelle ou originale. Au contraire, on retrouve un tel entêtement réformateur dans de nombreuses entreprises, publiques ou privées, françaises ou étrangères. C'est pourquoi, nous pensons que, d'une part, l'anonymisation de l'entreprise étudiée n'enlève rien à la compréhension du processus ; et d'autre part, que les interprétations et analyses proposées sont sans doute généralisables à de nombreuses situations.
} 


\section{II. - RECONSTITUTION \\ DE LA CONCEPTION MANAGERIALE DU CHANGEMENT}

\section{A / Précisions sur la notion de culture}

Mais avant, précisons le sens de la notion de culture, dont Bernard Valade (1992) a montré la polysémie. De manière générale, nous retiendrons que le terme désigne l'ensemble des conceptions et représentations, des images forces que l'on peut mettre en évidence chez les membres d'un groupe, ensemble qui préexiste aux situations que l'on observe. Par représentations, nous entendons les facultés mentales, les catégories collectives de pensée (Durkheim et Mauss, 1903). De plus, la culture est conçue comme une réalité construite et reproduite de génération en génération, ce qui permet de souligner qu'elle possède une homogénéité et que son évolution s'effectue lentement, sans nécessairement détruire les plus anciennes représentations ${ }^{1}$.

Dans le cas particulier de cette étude, notre ambition est de dégager, en analysant sa pratique, les représentations propres à l'identité culturelle des dirigeants. Celle-ci permet, dans différentes situations, d'engendrer des pratiques différentes; elle n'est pas a priori figée, mais sa plasticité lui permet de s'adapter à de nombreux domaines, sans avoir à changer ${ }^{2}$. Cette démarche est d'autant plus justifiée que le groupe dirigeant l'organisation étudiée, dont les membres appartiennent presque exclusivement à deux grands corps, possède des structures de formation-sélection parfaitement institutionnalisées et autonomisées, lui permettant de veiller à son propre renouvellement.

\section{B / Un corps social considéré comme peu adaptatif}

Venons en maintenant à la caractérisation des traits de culture soustendant l'action réformatrice. Toutes les transformations sont issues d'une volonté managériale, et les projets ont tous été conçus par un sous-groupe de l'état-major, même si certains l'ont été après une phase de recueil des opinions des salariés. Une constante de la pratique managériale est, malgré l'abondance des études commanditées par la direction, d'éviter de s'appuyer sur une connaissance fine des réalités du terrain.

\footnotetext{
1 “ Il n'est nullement certain que des parties considérables de notre mentalité ne soient pas encore identiques à celles d'un grand nombre de sociétés dites primitives (...) Est-on si sûr que même dans nos sociétés le pouvoir d'achat de la monnaie soit fondé en raison ?", DURKHEIM et MAUSS, 1903.

${ }^{2}$ En somme, la culture constitue “ un ensemble de schèmes fondamentaux, préalablement assimilés, à partir desquels s'engendrent, selon un art de l'invention, analogue à celui de l'écriture musicale, une infinité de schémas particuliers, directement appliqués à des situations particulières ” BOURDIEU, 1978.
} 
Au contraire, le management cherche à enseigner de nouvelles valeurs et pratiques : depuis le culte du service au client, jusqu'à l'acceptation de la compétition comme idéal de relation. Cette inculcation s'effectue d'ailleurs, non seulement par le suivi de stages et séminaires, ou par la lecture de supports de communication, mais essentiellement à l'insu des individus concernés, par la pratique répétée d'exercices de transformation de l'organisation. Cela suppose déjà que les dirigeants croient dans le bien fondé de modifier la culture des agents. Cela suppose aussi qu'ils sont persuadés que les agents ne sont pas capables de concevoir, par eux-mêmes, les comportements adaptés à leur situation.

On pourra rechercher l'origine de cette vocation pédagogique, dans le système de sélection-formation des élites, système fondé à la fin du XVIIIème siècle qui, comme nous allons le voir, s'inscrit dans la tradition ouverte par les utopies de Francis Bacon et Condorcet.

\section{C / Un corps social constitué d'une hiérarchie de particules}

Plus généralement, à travers la présentation de la vague de projets de changement qu'il conçoit, à travers le regard qu'il porte sur les initiatives délocalisées, par l'analyse des solutions qu'il privilégie, le haut management laisse transparaître la conception qu'il se fait du corps social de l'entreprise. Cette conception consiste à admettre que, pour être modelable à volonté, le social est constitué de "particules" élémentaires que sont, tantôt les individus ${ }^{1}$, tantôt les activités ${ }^{2}$, tantôt les entités organisationnelles de base ${ }^{3}$. Selon cette conception, il suffit de procéder à un recensement exhaustif de ces particules ${ }^{4}$, de leurs caractéristiques (liste de compétences), de les associer à un responsable et de leur communiquer une certaine quantité d'énergie (les connaissances et les promesses de reconnaissance), pour reconfigurer régulièrement leur assemblage.

Quant aux pratiques professionnelles qui lient entre elles ces particules, elles sont jugées réductibles à un ensemble fini de pratiques élémentaires (les modes d'emploi, les objectifs, les contrats), que l'on peut faire figurer sur un diagramme de flux en vue de leur simplification, avant automatisation.

L'analyse des réformes, notamment celle du mode de GRH, ainsi que la volonté d'introduire la contractualisation des activités internes, montre le poids de deux représentations: d'une part, la direction pense que les

\footnotetext{
${ }^{1}$ Les principes de la gestion du personnel introduisent une individualisation des modes de rémunération et des promotions.

${ }^{2}$ Les principes de l'organisation du travail découpent en tâches élémentaires les activités, en vue de leur simplification ou de leur recomposition. Le fonctionnement de l'entreprise est assimilé à une liste de processus et les métiers à des listes de compétences requises.

${ }^{3}$ Que l'on peut regrouper selon des combinaisons variées : fusion, absorption, délocalisation.

${ }^{4}$ C'est notamment ce que montre la manière dont l'élite envisage la réduction des effectifs.
} 
pratiques professionnelles sont entièrement objectivables a priori, sous la forme d'une liste de tâches; d'autre part, elle suppose qu'elle est capable de prévoir tous les chantiers à venir (des produits offerts, des métiers, des structures, etc.), comme s'ils ne faisaient que décliner en lots les objectifs plus généraux de la stratégie. Cette pratique se prolonge dans la volonté de maîtriser les processus de changement eux-mêmes, en " pilotant" la liste des réformes en cours, au sein d'une "sur-réforme", répertoriant chaque projet de changement comme une action.

\section{D / Un corps social aux mécanismes transparents}

Il découle de cette conception du social que l'élite conçoit idéalement son commandement comme centre clairvoyant et omniscient. En effet, toute réforme, même quand elle prétend partir de la connaissance des conditions d'exercice du travail, se présente comme une tentative de ramener l'organisation réelle à un schéma de fonctionnement transparent, lisible et contrôlable, du point de vue d'un superviseur ${ }^{1}$.

Une illustration récente de cette conception est fournie par les résultats d'une étude, datant de 1995, présentant l'entreprise en vingt "processus" ou regroupements d'activités majeures, déduites des rubriques de la comptabilité analytique. Chaque processus est réputé intégrer l'ensemble des tâches concourant à un même objectif. Plus précisément, un schéma de type systémique présente vingt rectangles, reliés entre eux par des flèches, formant une suite ordonnée partant du processus central "établir la stratégie". Le système est non bouclé : aucun circuit n'est prévu pour réguler la stratégie. Ainsi, depuis le lieu où la stratégie est élaborée, jusqu'aux tâches d'exécution, s'écoule idéalement une information non équivoque, non interprétable. Et la direction peut élaborer ses directives... sans se soucier de la bonne mise en oeuvre de ses précédentes directives.

De plus, cette mise à plat, obtenue à partir d'une connaissance purement comptable révèle une conception du social où l'ensemble des pratiques professionnelles sont conçues comme décorélées des finalités auxquelles elles contribuent : ainsi, la facturation d'un produit ou d'un service, existe indépendamment de la manière dont elle va être mise en oeuvre. Le fait d'automatiser (d'informatiser) la facturation est sans incidence fondamentale sur le fonctionnement du processus facturation. Une telle conception est sous-tendue par l'idée qu'il existerait des activités en soi, un modèle d'entreprise idéale, toute tendue vers la fourniture de services, par le biais d'une transformation de ressources.

On trouve une autre illustration de cette conception dans le choix des moyens de communication mis en oeuvre lors de la préparation de la loi de

\footnotetext{
${ }^{1}$ On retrouve la figure du Panopticon de J. Bentham (voir plus loin).
} 
privatisation. Un serveur télématique et une ligne directe permettaient de poser des questions, anonymement, et d'être informé, individuellement, sans bouger de son poste de travail. Chacun pouvait avoir le sentiment de parler au président lui-même, dans une sorte de tête à tête conservant l'anonymat. Le rêve du corps social transparent, où chaque membre, directement relié à l'état-major, puiserait les renseignements à la source, semble ici prendre forme ${ }^{1}$.

\section{E / Un corps social sans histoire}

Plusieurs éléments montrent que l'élite envisage l'histoire et la culture produite par l'expérience, comme un frein à l'efficacité collective. Ainsi se présente la succession des réformes. Chacune semble annuler l'héritage du "passé". De plus, chaque nouvelle réforme est présentée comme un moyen de résoudre des dysfonctionnements internes. Or, ceux-ci résultent, au moins en partie, de précédentes décisions et de précédentes réformes. Mais la responsabilité issue du passé n'est jamais soulignée. A cela s'ajoute une réforme particulière, qui sous-tend au fond toutes les autres, à savoir celle qui vise à inculquer une nouvelle culture. On y décèle bien une volonté de "faire du passé table rase", mais cette fois dans l'espace des représentations collectives. Notons que cette action dirigeante présente la particularité d'occulter le travail de construction des précédentes décisions.

D'autres illustrations de cette attitude an-historique sont fournies par la réforme des préretraites, ou encore l'incitation à la mobilité, et le remplacement systématique des outils par de nouvelles versions.

\section{F / Pratique modernisatrice et culture dirigeante}

En résumé, l'analyse de la pratique modernisatrice permet d'accéder la conception que l'élite se fait du social. Celle-ci, élaborée à partir d'une construction a priori et non d'une analyse du corps social ${ }^{2}$, est conçue comme connaissable, car composée d'éléments simples, eux-mêmes connaissables et donc hiérarchisables. Les salariés, ces composants élémentaires sont conçus comme facilement interchangeables ${ }^{3}$, incomplets et donc indéfiniment perfectibles au moyen de leçons pratiques les amenant à incorporer de nouvelles valeurs. La vocation didactique de l'élite s'en trouve

\footnotetext{
${ }^{1}$ On retrouve ici la pensée panoptique qui, loin de disparaître, persiste dans la croyance selon laquelle Internet, en mettant tout le monde en relation, va permettre de mieux communiquer. ${ }^{2}$ Il ne faut pas confondre le fait que la direction procède régulièrement à des audits, enquêtes et sondages, avec une volonté de comprendre le social. Les études commanditées visent à recenser les raisons d'un échec ou à anticiper sur des résistances, pour mieux les contourner. L'objectif de transformation est préalable à la volonté de connaître.

${ }^{3}$ Comme le prouvent les restructurations, la mise en préretraite, ou la réduction des métiers à des listes de compétences.
} 
renforcée, alors que ses membres ignorent l'essentiel des pratiques professionnelles. Derrière la posture du maître, on retrouve la volonté de contrôler, et notamment, de contrôler la bonne compréhension du savoir transmis, la bonne acquisition des valeurs, la bonne exécution des prescriptions.

Dès lors, l'organisation devrait être transparente, et l'échange s'effectuer sur le mode de la transmission d'informations non interprétables, unidirectionnelles et éphémères. Partant, les pratiques informelles et les identités professionnelles sont conçues comme des points de dysfonctionnement, des constructions le plus souvent irrationnelles, qu'il faut canaliser, supprimer ou contourner. On comprend, dans ces conditions que le changement, efficace pour l'entreprise, ne puisse provenir de l'organisation elle-même, mais de ceux qui voient clairement l'avenir. C'est pourquoi, la stratégie demeure une activité première et externe au corps social.

Puisqu'il s'agit d'un social abstrait, occultant l'existence de collectifs autonomes, on peut envisager de le former et de le déformer, au gré d'arbitrages rendus en fonction de règles d'efficacité ${ }^{1}$. L'oubli, produit par la succession même des projets de changement, permet de décoréler le verdict sur l'état présent, des décisions antérieures et ainsi, de limiter l'analyse des responsabilités. Mais surtout, il empêche la prise de conscience du caractère construit de l'organisation, ce qui la naturalise et permet de faire croire en l'existence de lois susceptibles de rendre compte de son fonctionnement. Dès lors, diriger c'est connaître et appliquer des lois.

Le tableau 1 présente cet arrière-plan culturel.

Tableau 1 : l'arrière-plan culturel des projets de changement

$\begin{array}{ll}\begin{array}{l}\text { Conception } \\ \text { de l'action }\end{array} & \text { Diriger, c'est connaître et appliquer des lois (clairvoyance) } \\ & \begin{array}{l}\text { Agir, c'est réformer, c'est-à-dire mettre en forme (toute- } \\ \text { puissance) }\end{array} \\ & \text { Les réformes sont envisagées } \\ & \text { - a priori, } \\ & \text { - à partir d'un modèle du corps social actuel } \\ & \text { - à partir d'un modèle du corps social futur } \\ & \text { - à partir d'un modèle du processus de transformation } \\ & \text { Le corps social, modélisable et modelable, est : }\end{array}$

\footnotetext{
${ }^{1}$ Il n'est pas jusqu'à la capacité d'inventer qui ne puisse faire l'objet d'un travail de planification, d'organisation, comme le montre l'existence d'un projet, dit "management des bonnes idées", et voulant inciter à la prise d'initiatives en prévoyant un taux moyen d'innovations par agent et par année.
} 
du corps social

- sans mémoire

- incapable de s'adapter de lui-même : $===>$ vocation pédagogique - réduit à un agrégat de particules : $===>$ vocation de clairvoyance

- constitué de relations objectivables : $===>$ vocation d'ordonnancement

Il est courant de rattacher la pratique managériale à la pensée taylorienne, soit pour en signaler la permanence, soit pour en montrer le dépassement. Dans cette perspective, l'arrière-plan culturel sous-tendant les réformes, tel que nous venons de le reconstituer, pourrait être utilisé pour souligner que certaines postures, défendues par Taylor, demeurent (conception a priori de l'organisation selon des critères techniques, corps social composé d'individus "stimulables", vocation pédagogique du management, recherche de transparence pour assurer l'harmonie sociale) ${ }^{1}$. Mais nous aimerions souligner une influence à la fois plus lointaine et plus puissante, car susceptible d'une certaine dynamique interne, celle qu'exerce, sur l'imaginaire managérial, le courant des utopies sociales (et plus particulièrement rationnelles). C'est dans cette perspective que nous allons chercher à rapprocher culture managériale et utopie rationnelle.

\section{III. - DEFINIR L'UTOPIE RATIONNELLE}

On peut considérer l'utopie comme un genre littéraire qui se serait dilué dans la science-fiction. On peut l'assimiler à une "vue de l'esprit", un projet de société irréalisable ${ }^{2}$ ou au contraire désirable (l'autogestion dans les années 1960-1970). Mais si l'on associe plus volontiers le thème de l'utopie à la philosophie politique ou à la littérature, la sociologie, depuis ses origines, y a vu, également, un objet d'analyse : Durkheim définit le socialisme en l'opposant à ce qu'il appelle l'utopie communiste (Durkheim, 1895-6) ; Michel Crozier aborde la question de l'utopie sociale en évoquant " le mythe de la transparence sociale" (Crozier et Friedberg, 1977); Alain Touraine propose une définition en la confrontant à l'idéologie (Touraine, 1965).

Nous ne nous intéressons toutefois qu'à un sous-ensemble du genre, celui des utopies rationnelles. Henri Desroche a proposé plusieurs systèmes de classification des textes utopiques. Dans « Humanisme et utopie » (1993), il utilise un découpage en "familles", selon l'angle privilégié de la réforme envisagée: "familiale, économique, ergonomique, écologique, andragogique, politico-scientifique, théologico-politique..." (p. 85), sans

\footnotetext{
${ }^{1}$ Taylor, Amar, Belot, Lahy, Le Chatelier, 1990.

${ }^{2}$ Engels et Marx reprochent au socialisme utopique de manquer de rigueur scientifique (ENGELS et MARX, 1844-1880).
} 
oublier l'éducation et la science. Mona Ozouf (1980) note que 1789 marque une rupture dans la production d'œuvres utopiques. Avant cette date, les utopies étaient essentiellement des outils intellectuels d'analyse du réel ; après 1789, elles ont contribué à influencer l'imaginaire des décideurs politiques, en leur fournissant des "images-forces". Pour Bronislaw Baczko (1978), de part et d'autre de la révolution, s'opère un "changement de paradigme" concernant l'utopie. Auparavant, le gouvernement parfait n'était considéré que comme une pure abstraction; après, les cités idéales " sont présentées comme autant de conséquences des théories sociales, comme des vérités scientifiquement fondées". Charles Rihs (1970) repère deux modes d'utilisation du discours utopique: l'un rationnel, positiviste, celui des Lumières ; l'autre " sentimental, populaire, romanesque ".

Prolongeant ces distinctions, pour caractériser l'utopie rationnelle, nous allons examiner, parmi le vaste ensemble des récits utopiques ${ }^{1}$, ceux qui : d'une part, entendent fournir un système rigoureux et cohérent de légitimation de la domination ${ }^{2}$; et qui d'autre part, ont eu une influence avérée sur le mode de sélection-production des dirigeants (inspirant la création d'un système de sélection-formation, elles ont été institutionnellement incorporées dans des pratiques). Sont particulièrement révélateurs de ce courant L'île d'Utopie (1516) de Thomas More qui a donné son nom au genre, ainsi que la Nouvelle Atlantide (1627) de Bacon et l'Esquisse d'un tableau historique des progrès de l'esprit humain (1794) de Condorcet, qui ont eu une influence sur l'élaboration d'un nouveau système de formation pour l'élite technicienne. Par ailleurs, La physiologie sociale (1808) de Saint-Simon présente la particularité, non seulement de donner à la pensée rationalisatrice sa forme utopique contemporaine, mais également d'avoir été rédigée par l'un des premiers bénéficiaires de ce système. Enfin Le panoptique (1791) de Jérémie Bentham fournit une exacerbation de cette forme. Ainsi situées, les utopies rationnelles manifestent une cohérence propre: elles constituent "le discours d'un groupe et non une oeuvre littéraire flottant en l'air" "3.

\footnotetext{
${ }^{1}$ Henri Desroche note que les textes écrits par les utopistes sont si nombreux qu'une " bibliographie, même signalétique, (...) occuperait à elle seule tout un volume " (DESROCHE, 1993).

${ }^{2}$ Comme le souligne Paul Ricoeur (1986), l'utopie traitant de la meilleure forme de gouvernement, elle rencontre nécessairement la question de l'autorité. Mais toutes ne développent pas un travail de légitimation de la domination, à partir d'un système cohérent de justification.

${ }^{3}$ RICOEUR, 1986, p. 361.
} 


\section{A / Gouverner selon les lois de la nature}

De L'île d'Utopie ${ }^{1}$, il convient de retenir deux caractéristiques qui seront reprises par la plupart des philosophes utopistes. Tout d'abord, l'ouvrage est découpé en deux parties. La première comprend la critique de l'existant (la société anglaise du XVIe siècle), critique qui symbolise le refus de la résignation et constitue un encouragement à l'activité imaginaire. La seconde partie comprend la description du modèle substitutif, âge d'or égalitaire dont la vie quotidienne est parfaitement réglée. Cette opposition entre les deux parties est didactique : elle montre, dans la mesure où la cité est " totalement et radicalement de l'homme ", que l'on peut conduire une action sans l'aide d'aucune transcendance ${ }^{2}$. Par ailleurs, il fonde la cité idéale (ou cité nouvelle) sur une conception humaniste du droit naturel. Selon cette conception, pour être justes, les lois humaines doivent être le reflet d'une loi de la nature, d'ordre divin. Ainsi, par le biais d'un raisonnement de juriste, More, ouvre la voie aux futures utopies qui se réclameront également de la nature, mais sous la forme de lois déduites de l'observation.

Dans La nouvelle Atlantide ${ }^{3}$, Francis Bacon a proposé une réforme de l'enseignement et de la recherche. Critiquant les universités où les étudiants “ n'y apprennent qu'à croire ; d'abord à croire que d'autres savent ce qu'euxmêmes ne savent pas, puis à croire qu'ils savent ce qu'ils ne savent pas " (p. 13), Bacon prône une recherche pure, indépendante de tout pouvoir (politique ou religieux) et s'appuyant sur l'observation de la nature, mais "sans avoir toujours le regard rivé sur l'utilité immédiate" (p. 14). Il souhaite ré-former le savoir théorique, en lui donnant des bases empiriques. A l'opposé de More, Bacon affiche sa foi en un progrès illimité, s'appuyant sur une méthode pour systématiser le processus de recherche (techniques d'observation, laboratoires spécialisés). Il est en effet le premier à appliquer les principes de l'analyse empirique à l'organisation de l'université et de la recherche, en introduisant une nette division du travail.

Par ailleurs, le progrès de la connaissance n'est pas gratuit, il a pour fin " de connaître les causes, et le mouvement secret des choses ; et de reculer les bornes de l'Empire Humain en vue de réaliser toutes les choses possibles" (p. 119). Cette intervention sur la nature s'apparente au prototype de la science de l'ingénieur, ni totalement abstraite (même si elle s'appuie sur un savoir théorique étendu), ni totalement empirique (elle serait alors un simple savoir-faire ouvrier, artisanal). Quant à l'utopie politique associée,

\footnotetext{
${ }^{1}$ En réalité, le titre original en latin peut être traduit par Le livre très précieux et non moins salutaire que plaisant de la meilleure des républiques et de la nouvelle île d'Utopie, SIMON, 1991, p. 97, note 1.

${ }^{2}$ LACROIX, 1994, p. 90-98.

${ }^{3}$ BACON, 1627.
} 
Bacon nous laisse penser que le bonheur social est supposé résulter d'une organisation réussie du système scientifique : les savants ne doivent être soumis à aucun contrôle démocratique et, en ce sens, sont les maîtres ultimes de la cité.

Finalement, si la science est aussi conçue comme une critique de la soumission à toute autorité hétérogène, elle contient le risque d'une confiscation des décisions au profit de l'élite savante, cooptée.

\section{$B$ / Réformer le système pédagogique}

La pensée de Bacon influença, directement par ses thèses, celle de Condorcet qui, selon Henri Desroche, ne cesse " d'entretenir en lui et autour de lui la nostalgie de cette Nouvelle Atlantide, par lui, quelque peu remathématisée "1. Mais il fut également influencé indirectement, par les premières tentatives de mise en œuvre de l'utopie baconienne. Nous pensons ici à D'Alembert, qui lui permit d'élaborer un modèle idéal d'organisation rationnelle de la science. Il fréquenta la haute fonction publique «éclairée ${ }^{2}$, qui cherchait à mobiliser la bureaucratie de la monarchie pour donner aux problèmes politiques des réponses rationnelles. Plus généralement, note Alexandre Koyré, nous retrouvons chez Condorcet " les idées de Voltaire, de Rousseau, de Turgot, d'Helvétius, de Condillac », peu à peu façonnées dans un tout harmonieux dont la dernière expression est l'Esquisse d'un tableau historique des progrès de l'esprit humain, "sorte de résumé philosophique du XVIIIe siècle "3, où réside le cœur utopique de la pensée révolutionnaire. Condorcet la rédige pour " montrer, par le raisonnement et par les faits, qu'il n'a été marqué aucun terme au perfectionnement des facultés humaines ; que la perfection des facultés humaines est réellement indéfinie "4. Il nous livre en réalité, moins un tableau des progrès, que la liste des erreurs dont l'humanité dut triompher. Il découpe ainsi l'évolution passée en neuf époques (partie critique), et leur oppose une dixième de son invention, l'avenir (programme scientifique de maîtrise de la nature et des mécanismes sociaux). Par sa structure, l'œuvre s'apparente ainsi aux utopies.

Mais Condorcet fait subir une distorsion au mode utopique. On notera en effet, que la preuve de l'existence d'une tendance naturelle au progrès s'appuie : d'une part, sur un passé fictif, une histoire sélective, taillée "sur mesure" pour les besoins de sa démonstration; et d'autre part, sur un avenir

\footnotetext{
${ }^{1}$ DESROCHE, 1993, p. 114.

${ }^{2}$ Turgot (1727-1781), intendant des Finances, et Trudaine de Montigny (1703-1769), administrateur du Corps des Ponts et Chaussées.

${ }^{3}$ KOYRE, 1971, p. 103.

${ }^{4}$ CONDORCET, 1793, p. 81. C'est Turgot qui a exposé, le premier de façon claire, la thèse de la solidarité des différents progrès accomplis par l'humanité, dans Le Tableau philosophique des progrès successifs de l'esprit humain, 1750.
} 
forcément inventé. e raisonnement est circulaire : pour prouver que le progrès existe, l'auteur décrit un avenir radieux, et le présente comme la résultante, à la fois logique et en rupture, du passé. En d'autres termes, le perfectionnement indéfini du genre humain " est indémontrable autrement que par son extrapolation sur l'avenir, c'est-à-dire, autrement que par la vision utopique d'une société autre "1.

Sa conception du bien public a pour contrepartie d'envisager les réactions populaires, les foules revendicatives, les émeutes sociales comme irrationnelles et justifie rationnellement la dictature ${ }^{2}$. Attitude que l'on retrouve dans l'inflexibilité avec laquelle furent mises en oeuvre les réformes, entreprises par Turgot et les Physiocrates, pour rendre le marché transparent. Il en va de même de la volonté de Condorcet d'édifier une science sociale, basée sur l'observation et l'accumulation de données statistiques, dont le but serait d'aider à "faire passer" la Raison en comprenant mieux les réticences du corps social, la persistance des "superstitions" et "traditions". La pensée rationnelle semble alors incapable d'imaginer que la Raison puisse émerger du social.

Enfin, comme le rappelle Alain Pons ${ }^{3}$, la fondation de l'Ecole Polytechnique a été directement inspirée par les idées de Condorcet sur la recherche scientifique. Il fut, de plus, rédacteur du " projet de législation sur l'instruction publique, le plus remarquable et intellectuellement le plus solide parmi les centaines de textes produits pendant la période révolutionnaire "4. Ainsi, Condorcet a non seulement fait évoluer le genre utopique en s'appuyant sur l'œuvre (intellectuelle et pratique) de ses prédécesseurs, mais sa propre utopie a reçu très tôt une première mise en œuvre.

\section{C/ Réformer la société grâce aux entrepreneurs}

La vie et l'œuvre du comte de Saint-Simon constituent un trait essentiel entre les premières esquisses de la rationalité techniciste et sa traduction dans de nombreux domaines (notamment, la gestion) ${ }^{5}$. Pierre Musso (1997) rappelle les différents tournants de la vie du comte : il participe à la guerre

\footnotetext{
${ }^{1}$ BACZKO, 1978, p. 202. Cette pratique sera couramment reprise, notamment par SaintSimon (voir ci-dessous), puis par Etienne Cabet qui élaborera un " tableau des progrès de la démocratie ", pour montrer que le projet utopique proposé est non seulement réaliste, mais répond à une tendance naturelle.

${ }^{2}$ Nicole S. Morgan décèle, dès l'Utopie de T. More, cette tendance. En effet, comme “ tout le monde travaille de son mieux et pour le plaisir de chacun et de tous (...) l'improductivité devient le péché ", ce qui " octroie aux Utopiens un droit naturel d'annexer toute terre dont ils ont besoin et qui est mal gérée par les indigènes". MORGAN, 1996.

${ }^{3}$ Qui préface l'édition de l'Esquisse, aux éditions Garnier Flammarion, 1988.

${ }^{4}$ Une éducation pour la démocratie, 1982, Textes et projets de l'époque révolutionnaire, recueillis et présentés par BACZKO (B.), Garnier, p. 9.

${ }^{5}$ FRICK, 1988.
} 
d'indépendance en Amérique; suit une formation d'ingénieur de l'Ecole royale du génie de Mézières; étudie les canaux en Hollande; devient entrepreneur ; fréquente les Ecoles Polytechnique et de médecine. Il n'a pu manquer d'adhérer aux discours dominants sur les sciences et aux logiques qui les sous-tendent. Certes, Saint-Simon reproche à Condorcet d'avoir oublié l'importance des relations de pouvoir dans le fonctionnement du social ${ }^{1}$ et d'avoir pensé que les religions ont été des obstacles au développement des sociétés, mais il reconnaît en lui un précurseur en matière d'étude rationnelle du social.

D'ailleurs, tout comme Condorcet, Saint-Simon utilise l'analyse du passé pour montrer que le processus de progrès social est déjà engagé. Mais à l'encontre de son prédécesseur, il met l'accent sur " la lutte qui a toujours existé entre les organes du corps social, entre les chefs et les administrés "2. C'est pourquoi il cherche à dégager de l'observation du passé les lois $d u$ changement social, du monde moral et du monde politique. Pour y parvenir, Saint-Simon entreprend "d'unifier l'ensemble des sciences (physiques et humaines)" en reformulant "la loi de la gravitation universelle afin d'en faire la loi d'ensemble des êtres", tant physiques que sociaux. Dès lors, le nouveau système philosophique, adapté à la société du XIXe, découle de la nature même de cette société. Celle-ci, caractérisée par un essor démographique et économique sans précédent, doit être gérée par "la science de la production, c'est-à-dire la science qui a pour objet l'ordre des choses le plus favorable à tous les genres de production " $"$.

1 élabore un système qui " rend anachronique toute forme de conquête ou de domination ". Dans ce système, le travail est le ciment social, à condition de l'organiser rationnellement, c'est-à-dire de laisser décider les industriels (représentant de tous les travailleurs). Comme le résume Durkheim, à la tête du système social qu'il prône, les membres du conseil industriel " ne disent pas d'ordre, ils déclarent seulement ce qui est conforme à la nature des choses. Les savants démontrent quelles sont les lois de l'hygiène sociale, puis, parmi les mesures qu'ils proposeront, en conséquences de ces lois, les industriels choisiront $" 4$. Sa conception de la rationalisation du social consiste à prôner le renouvellement des élites et leur formation, en

1 “ Tout homme, toute coalition d'hommes, de quelque nature qu'elle soit, tend à accroître son pouvoir ". SAINT-SIMON, 1965, p. 49. Extrait de L'introduction aux travaux scientifiques du XIXe siècle, 1808.

${ }^{2}$ SAINT-SIMON, 1965, p. 60. Extrait de De la physiologie sociale, 1813.

${ }^{3}$ SAINT-SIMON, 1965, p. 78. Extrait de Lettres à un Américain. Si Saint-Simon prolonge More et Bacon, qui tous deux avaient pensé l'organisation sociale du travail, il influença à son tour les concepteurs d'utopies. C'est ainsi qu'Etienne Cabet remplacera, à la tête de son Icarie, la noblesse par l'élite des professions.

${ }^{4}$ DURKHEIM, 1895-6, p. 166. 
appliquant à la sphère du pouvoir, les principes déjà éprouvés dans les sciences de l'ingénieur.

Saint-Simon a la particularité d'avoir été parmi les premiers à connaître l'influence des utopies rationnelles, telles qu'elles ont commencé à se déployer dans le fonctionnement de l'institution de Polytechnique et des écoles de médecine. Il a été également l'un des premiers à se saisir de ces connaissances pour les perfectionner et les diffuser à nouveau sous forme d'utopie, en adjoignant le mythe du travail à celui du progrès (Ricoeur, 1986). Ce qui montre combien la pensée utopique est dynamique et tient compte des acquis de son temps.

L'idée de placer l'entrepreneur au cœur de la réforme sociale, se retrouve chez un auteur dont l'œuvre, par sa densité et sa logique interne montre à quel point la pensée rationaliste avait diffusé dans le corps social au XIXème. Nous pensons ici à Jeremy Bentham (1748-1832), théoricien de l'utilitarisme, du libéralisme économique et concepteur d'un modèle d'organisation universel. Dans le Panoptique, l'auteur propose en effet un plan-type de bâtiment pouvant servir à plusieurs usages: " punir les criminels endurcis, garder les fous, réformer les vicieux (...) employer les oisifs (...) ou dispenser l'instruction aux générations montantes "1. Il décrit le mode d'administration de cet édifice polyvalent, dont il envisage de confier la direction à un gérant, payé en fonction de l'atteinte de ses objectifs (vies humaines épargnées dans le cas des prisons).

Dans ce cas aussi, l'auteur effectue ses propositions pour améliorer une situation catastrophique, celle de l'état sanitaire des prisons du XVIIIème siècle. Son projet est sous-tendu par une volonté d'éduquer (ou de rééduquer) les pensionnaires de son pénitencier-usine : provoquer, par " une soumission forcée ", " une obéissance machinale ". Là également tout est précisé : type de travail, de nourriture, de vêtements, les consignes pour maintenir l'hygiène et l'emploi du temps des loisirs. Plusieurs innovations technologiques sont introduites, aussi bien pour améliorer (à moindre coût) les conditions d'hygiène des pensionnaires, que pour démultiplier les capacités de surveillance à distance.

S'il inspira James Mill et J. Stuart Mills, la publication de son Panoptique, en France, "sur ordre de l'Assemblée nationale", montre que ses conceptions entraient en écho avec celles des Lumières, telles qu'elles ont été traduites chez les Ingénieurs, les Physiocrates, les réformateurs sociaux: transparence, fluidité des échanges, circulation de l'air et des capitaux, éducation des masses.

$D$ / Esquisse d'une définition de l'utopie rationnelle

${ }^{1}$ BENTHAM, 1791, p. 98. 
Ainsi, les œuvres analysées ont en commun de se référer à la science : leur projet de société est justifié à partir d'une analyse scientifique du passé ; les dirigeants sont sélectionnés et formés sur des critères scientifiques; l'imagination, les représentations sont réglées selon une logique scientifique.

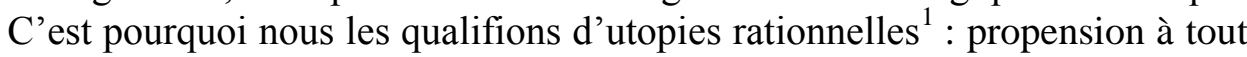
ramener à un principe déduit de la nature ; croyance en l'existence de lois préexistantes; volonté de fonder une science (le calcul mathématique des destinées) ; organisation minutieuse du travail et des loisirs, importance de l'arithmétique. On peut, dès lors, dégager trois registres de sens qui délimitent une définition de ce concept.

A/Le premier concerne le résultat d'une dynamique de création intellectuelle, s'étageant sur plusieurs siècles, reflétant le point de vue d'un groupe (souvent dominant dans le champ intellectuel) et interagissant avec les conditions sociales et politiques. Existe ainsi un véritable phénomène d'apprentissage de la pensée utopiste qui, notamment à travers les tentatives d'expérimentations (saint-simoniens, fouriéristes, cabétistes, owenistes, mais aussi, plus discrète et efficace, la création de l'Ecole Polytechnique comme prolongement de l'œuvre de Condorcet), n'abandonnent jamais le projet utopique de conformer le social à un plan préétabli. Ce que l'on peut résumer ainsi: "le rêve utopique est un rêve fait de trames ou de scénarios constamment réécris, repeints, remaniés à chaque génération " (FISCHER, 1993).

Si elles proposent des solutions sociales globales, elles se présentent sous la forme de récits scindés en deux : une critique de l'existant, suivie de la description d'un idéal. Ces deux dimensions sont liées, puisque la critique n'est fondée que si l'on parle depuis la cité idéale, de l'autre côté de la rupture (logique ou temporelle). C'est donc définir l'utopie par sa méthode, " très proche de tous les procédés ordinaires d'invention scientifique : méthode hypothético-déductive, et expérience mentale" (RUYER, 1950, p. 11).

$\mathrm{B} / \mathrm{A}$ un second niveau, l'utopie peut être conçue comme un ensemble d'images-forces, impressionnant les croyances collectives, et s'intégrant au

\footnotetext{
${ }^{1}$ A contrario, toutes les utopies ne sont pas rationnelles, même si l'on trouve souvent une tendance à rationaliser le mode de commandement, y compris dans des œuvres que l'on considère comme libertaires. On pense ici à Fourier, qui a placé au centre de son dispositif la libre satisfaction des passions. En réalité, son but est de trouver une articulation entre passions et mode de gouvernement. Pour cela, il critique les conséquences de la société industrielle naissante, en se référant, lui aussi, à un principe déduit de la nature. Il s'agit du principe d'attraction, introduit par analogie à l'attraction newtonienne, et qui serait à la source de toutes les passions. La société idéale qu'il promeut nécessite, en arrière fond, l'abondance matérielle, obtenue par un nouvel ordre industriel. Pour rendre compatible passions et productivité, il envisage de rendre obligatoire la rotation dans les tâches et encadre très précisément les activités quotidiennes (on rencontre chez lui une sorte d'obsession des nombres).
} 
corps d'idées influençant l'élite. Cette précision n'est pas neutre, car “ les images possèdent une force. Par elles, l'utopie suscite l'élan enthousiaste ou creuse l'insatisfaction (...) L'image engage un mouvement de la pensée "1. Voilà qui explique la force spécifique de l'utopie: par ces images, elle séduit, provoque et déclenche un processus cognitif, d'autant plus puissant qu'il se présente comme libérateur et progressiste.

On notera que ce qui est ainsi proposé à l'imagination n'est pas, à proprement parler, une création, une invention, car "l'utopiste fait de l'ailleurs et de l'avenir avec de l'ici et du maintenant (...) en faisant fonctionner, un peu de travers, ce qui existe déjà "2. Mais "l'utopie veut installer la raison dans l'imaginaire ", de facteurs qui organisent l'ensemble des idées et des sentiments "4. Il en résulte que les détails de la cité future, sur lesquels insistent tant les concepteurs d'utopie, loin d'être extravagants, constituent autant d'applications strictes des principes généraux organisant la société globale. Ici, le local incarne, répète et enseigne le global.

C'est dans l'espace imaginaire que les utopies peuvent être "tirées" vers l'idéologie. D'ailleurs, Paul Ricoeur note que l'on ne peut comprendre l'utopie sans prendre en compte l'idéologie. En effet, si l'on admet que ce qui caractérise le discours idéologique, c'est qu'il est " non congruent" avec la réalité sociale, dès lors, en tant qu'il explore le possible, il nous place en marge du réel, et en cela construit un lieu d'où porter un jugement distancié, extérieur: "le jugement porté sur une idéologie l'est toujours depuis une utopie ". L'utopie est alors ce qui permet de dénoncer comme idéologique toute représentation ou discours, produit pour conférer aux gouvernants le surplus de légitimité qui lui faisait défaut.

$\mathrm{C}$ / Enfin, troisième niveau, le modèle de cité peut être conçu comme un guide détaillé pour l'action, dans la mesure où il s'appuie sur une conception du social comme transparent et intégralement accessible à la connaissance. Mais cette institutionnalisation du modèle n'est pas sans conséquence : réaliser l'utopie, c'est arrêter le temps et instaurer le conformisme car, pour assurer la stabilité du monde parfait, il faut évacuer toute conflictualité (Paul Ricoeur parle de l'obsession de la non-contradiction). Ce qui peut nécessiter l'exercice d'un contrôle social oppressant. De plus, l'expérimentation étant réalisation du bonheur, toute critique ne peut qu'être dénoncée comme irrationnelle, et doit être soumise à rééducation. En conséquence, dès lors

\footnotetext{
${ }^{1}$ THOMAS, 1997.

${ }^{2}$ RUYER, 1950, p. 57.

${ }^{3}$ BACZKO, 1978, p. 32.

${ }^{4}$ RICOEUR, 1986, p. 361.

${ }^{5}$ RICOEUR, 1986, p. 231.
} 
que le projet de cité idéale devient plan à mettre en oeuvre, certains de ses traits sont exacerbés : symétrie («l'utopie consiste à traiter les problèmes comme des problèmes d'architecture et d'urbanisme ${ }^{1}$ ), uniformité (élimination de la dissidence, de l'imprévu), croyance aux vertus de l'éducation, hostilité à la nature, dirigisme, ainsi que le sentiment de toute puissance et l'an-historisme.

Tableau 2. Les différents niveaux du concept d'utopie

Plan de la créationProcessus historique, fait progresser la connaissance

Intellectuelle

et de la méthode

Elle est proche de la méthode hypothético-déductive

Exerce la force créatrice des hypothèses.

Plan de l'imaginaire des croyances

Plan de la pratique (utopie réalisée)
Le modèle idéal est un complexe d'images-forces, cherchant à rendre cohérent l'imaginaire.

Il est composé de croyances telles que :

- valorisation de la volonté ;

- le progrès est dans la nature des choses ;

- l'opinion publique n'est qu'ignorance (élitisme) ;

- les hiérarchies sociales s'appuient sur des hiérarchies de savoirs ;

- il faut rompre avec les traditions.

Présente le risque de dérive/récupération idéologique.

Peut s'enrichir et interagit avec le réel

L'idéal est un guide détaillé pour l'action, basé sur une connaissance scientifique du social.

Une action est rationnelle si elle est précédée de l'élaboration d'un plan (but). Ce qui valorise la figure de l'homme d'action.

La mise en oeuvre requiert l'absence de conflits et ignore la dialectique.

${ }^{1}$ RUYER, 1950, pp. 41-44. 
Elle implique : an-historisme ; conformisme ; normalisation ; méconnaissance des effets secondaires et composés ; renforcement du sentiment de toute puissance.

Conduit à transformer le modèle originel, ou à basculer dans le totalitarisme

Il est alors clair que l'utopie ne se confond pas strictement avec l'idéologie (elle peut y recourir, s'y confondre momentanément, mais elle ne s'y réduit pas). Il n'est pas, non plus, pertinent d'y voir une forme de mythe, ce type d'explication avancé par la société sur ses origines, son fonctionnement, celui de la nature et reconnu pour vrai par les membres de la société : tout au plus peut-on dire que l'utopie peut s'inspirer de la mythologie et qu'elle peut concourir à forger de nouveaux mythes ${ }^{1}$. Enfin, c'est le plan de la création intellectuelle, susceptible d'enrichissement (à l'horizon non prévisible), qui distingue l'utopie rationnelle de l'ingénierie sociale $^{2}$, cette dernière se confondant essentiellement avec le niveau du guide détaillé pour l'action (il ne s'agit pas de changer la société, mais de la faire fonctionner sans heurt), tout en possédant, certes, une dimension imaginaire, mais réduite à son versant manipulatoire, idéologique ${ }^{3}$.

Mais plus fondamentalement, quel peut être l'apport d'un tel outil d'analyse pour comprendre la pratique réformatrice en entreprise ?

\section{$I V$. - Utopie rationnelle et culture réformatrice : quelques identités}

Il ne s'agit pas de tomber dans le travers de la modélisation, et d'appliquer à la complexité de l'action managériale, une grille d'analyse idéalisée à outrance. En effet, le passage de la réflexion littérale à sa condensation sous forme d'énoncé lapidaire, ne doit pas masquer la réduction

\footnotetext{
${ }^{1}$ Pour une discussion de la définition des mythes, voir SMITH (Pierre), 1995, article " Mythe ", Encyclopaedia Universalis.

${ }^{2}$ Le terme désigne une doctrine, encore appelée "génie sociologique", fondée par les disciples de Le Play (notamment Emile Cheysson), dans la postérité de Saint-Simon (et de Condorcet), et qui consiste à introduire un nouvel acteur, l'ingénieur social, chargé d'instaurer la paix sociale, en doublant l'ingénieur technique d'un expert du social, soucieux des conséquences sociales du fonctionnement des entreprises. A noter que ces experts de l'organisation sociale sont, selon Le Play, à choisir de préférence parmi les chefs d'entreprise que la complexité de leur métier est supposée tout particulièrement préparer à la compréhension des mécanismes sociaux (KALAORA et SAVOYE, 1989). Appliquée à l'entreprise (et plus généralement aux organisations), l'ingénierie sociale peut prendre la forme de la doctrine taylorienne, accueillie avec enthousiasme par les ingénieurs, notamment polytechniciens (H. Le Chatelier).

${ }^{3}$ Comme le montre, par exemple, l'œuvre et la vie de F. W. Taylor, mêlant aux principes dérivés des méthodes d'ingénierie, la recherche d'une harmonie sociale (« le refus de la lutte des classes au profit d'une coopération arbitrée par les experts »(POUGET, 1998, p. 70).
} 
opérée, notamment en oblitérant les relations dynamiques qui s'exercent entre la dimension imaginaire de l'utopie et l'environnement social dans lequel vivent les auteurs d'utopies; ainsi qu'entre la conception du changement et les différentes phases de sa mise en oeuvre. C'est pourquoi, nous allons souligner les dimensions du phénomène que cet outil d'analyse permet d'éclairer.

- Au niveau de la dynamique intellectuelle

On peut noter, en premier lieu, la proximité entre certaines dimensions du concept et certains traits de culture des dirigeants, au niveau de la production de principes, de méthodes, de cadres d'analyse.

* Les concepteurs de réformes, tout comme les dirigeants des Cités idéales, sont sélectionnés et formés sur des critères d'excellence scientifique et technique ;

* Tous les deux conçoivent le social comme connaissable: a) par décomposition en éléments simples (individus, services, compétences, fonctions, voire traits de caractères, etc.) ; b) et par identification des règles d'agencement-agrégation entre ces éléments simples ;

* Tous les deux conçoivent le social comme contrôlable et déformable pour le rendre harmonieux, transparent et cohérent.

- Au niveau des croyances et de l'imaginaire

Les concepteurs de réformes, tout comme les dirigeants des Cités idéales, sont persuadés que l'on peut et que l'on doit conformer le social relativement à un plan logique préétabli et établi sans les principaux intéressés (ou en ne tenant compte de leur opinion que de façon instrumentale, manipulatoire, pour mieux les contourner ou s'en servir de levier).

Le plan de la Cité ou le projet de réforme, en tant que modèles a priori, prétendent : répondre à des dysfonctionnements et avoir été élaborés par application de principes rationnels (connaissance des lois du réel).

Dans les deux situations, on cherche à produire de l'adhésion, de la conviction et pour cela, tout vise à réformer la culture : en utopie, «par une éducation totale » pour éradiquer la superstition et produire des citoyens ; et, dans les entreprises, pour briser les résistances au changement afin de moderniser.

- Au niveau de l'action-expérimentation

* Dans le cas des projets de Cité idéale, l'action peut déboucher :

- soit sur le totalitarisme, si le réformateur prend au pied de la lettre le projet ;

- soit sur des expérimentations limitées, susceptibles d'être abandonnées, qui peuvent donner naissance à de nouvelles utopies (ceux qui ont mené l'expérience écrivent à leur tour «leurs mémoires », sous forme de nouvelles utopies). 
Il en résulte une évolutivité du «genre utopique », par enrichissement et intégration de nouveaux matériaux.

* Dans le cas des projets de réformes, le scénario est très semblable, puisque au moment de la mise en œuvre :

- soit les injonctions sont prises au pied de la lettre, avec zèle, et dans ce cas, on rigidifie les structures ainsi que le fonctionnement ;

- soit on procède par expérimentations successives, et le groupe dirigeant apprend à maîtriser de nouveaux auxiliaires du changement, en instrumentalisant de nouveaux domaines de connaissances (formation, information, technologies) et des groupes sociaux (promotions par cooptation, utilisation des clivages entre syndicats).

En somme, l'identité culturelle du groupe dirigeant est structurée autour d'une vision utopique du social et de son rôle, cette vision lui permettant de se préserver, tout en disposant d'instruments de renouvellement et de moyens de produire l'adhésion.

On peut, en prolongeant le raisonnement, penser que, si le concept d'utopie permet de rendre compte de la volonté managériale de changement et de la forme de son action, c'est parce que ce groupe a pu s'identifier au projet de société porté par le courant des utopies sociales, tel qu'il a été formulé à la fin du XVIIIème siècle. En effet, les utopistes ou les réformateurs sont d'autant plus amenés à persévérer dans leur projet, que ce dernier est redoutablement cohérent. Tout se passe comme si ce qu'il y a de plus essentiel dans ce courant (illustré par l'idéal rationaliste et rationalisateur de Condorcet), en s'incarnant dans plusieurs institutions (grandes écoles, grands corps, hiérarchies sociales fondées sur des distinctions scolaires) ${ }^{1}$ et rejaillissant ultérieurement en de nouvelles utopies (de celles de Saint-Simon aux discours réformateurs, en passant par l'accueil fait aux thèses de Taylor), avait peu à peu colonisé le monde vécu (Habermas), d'abord des membres de l'élite puis, progressivement, de l'ensemble du corps social.

\section{CONCLUSION : UTOPIE ET COMPREHENSION DE LA PRATIQUE MANAGERIALE}

Mais, plus qu'un élément de l'histoire des idées, le concept d'utopie rationnelle permet de rendre compte en un seul mouvement de l'articulation entre le système culturel managérial, la pratique de la réforme permanente et leur interaction (source potentielle d'apprentissage). En effet, les utopies ne surgissent pas ex nihilo, elles sont élaborées par des membres de l'élite,

\footnotetext{
${ }^{1}$ L'interaction entre ces différents acteurs se dégage de la lecture de textes concernant la biographie de Condorcet (BAKER, 1975), l'histoire des grands corps techniques (PICON, 1992) et celle des grandes écoles (DHOMBRES, 1987).
} 
soucieux de condenser, en une formule aisément communicable, l'essentiel des idées réformatrices que leur a inspiré leur expérience (souvent faite de la difficulté à expérimenter - jusqu'au bout - l'utopie de leurs prédécesseurs). Et ces "synthèses" influent sur les futures tentatives de réformes, grâce aux nouvelles images-forces qu'elles incorporent au stock préexistant.

De plus, l'accès à l'imaginaire situe le lieu où s'effectue le retournement de la critique en défense du statu quo (idéologie), légitimant l'action. On peut donc tenir en un seul mouvement, volonté de réforme et domination (ou volonté de domination), exercée par le groupe concepteur du projet. En effet, de par sa logique même, qui consiste à vouloir (re)construire, sans cesse, les structures de l'organisation, l'utopie-expérimentation renforce le rôle et les positions de pouvoir des experts du changement. La question de la légitimité est alors naturellement intégrée au corps de concepts.

On peut aussi repérer comment s'effectue l'inversion du projet de réforme en source de rigidification: dès lors que le plan est pris pour un guide détaillé de l'action, qu'il entend tout organiser, y compris la culture, et qu'il mobilise tous les moyens pour y parvenir, il ne peut s'adapter et tend à se répéter.

Sous un autre angle, les discours utopiques sont d'autant plus nombreux et mobilisés par les acteurs sociaux, que la croyance en la possibilité de changer le monde est renforcée. Dès lors qu'une rupture advient empiriquement (comme lors d'un événement historique réputé majeur), la tentation est grande d'expérimenter les projets de cités idéales. L'influence de l'environnement n'apparaît plus alors comme un paramètre secondaire, mais comme une dimension structurante. On comprend simultanément qu'en période de disparition des repères, un ordre peut s'instaurer : il s'effectue en référence à un substrat culturel protégé parce que profondément ancré (avant l'entrée dans l'organisation et conforté par une multitude d'institutions et de projets aboutis).

Ainsi, à la rationalité limitée et au mode de décision anarchique (modèle de la poubelle), il nous semble pertinent d'adjoindre l'utopie rationnelle (le cadre cohérent d'explications qu'elle fournit), pour analyser la réforme permanente des entreprises («modernisation »). En effet, l'intérêt du détour par la notion d'utopie rationnelle est de montrer qu'il existe une évolutivité intrinsèque des pratiques réformatrices, dont la rationalisation des procédures de changement permanent n'est que l'étape contemporaine. C'est ce lent apprentissage dont rend compte le concept ici forgé, apprentissage qui débouche sur un perfectionnement, non de l'efficacité des systèmes organisés, mais de la capacité à réaliser un programme. Ce progrès a été permis, dans l'exemple analysé, par le recours à toutes sortes d'"auxiliaires", depuis la rationalisation de la formation et de la GRH, jusqu'à la 
mobilisation de salariés intermédiaires, en passant par l'introduction de systèmes techniques sophistiqués.

On peut supposer que, du point de vue managérial, surviendront des perfectionnements dans cette voie. Si l'on peut craindre avec Georges Duveau, « qu'en se livrant à des essais de géométrie non-euclidienne, (...) [les managers] ne fabriquent des monstres ${ }^{1}$, on peut également voir dans la dynamique même de l'utopie rationnelle, par l'interaction entre pratique et imaginaire, le lieu d'un dépassement toujours possible.

France Télécom Recherche et Développement

38 rue du Général Leclerc

92000 Issy-les-Moulineaux

(Associé au LSCI-ERESMO, 59 rue Pouchet, 75017 Paris)

\section{BIBLIOGRAPHIE}

BACON F., 1627, La nouvelle Atlantide, GF- Flammarion, 1995.

BACZKO B., 1978, Lumières de l'utopie, Payot.

BAKER K. M., 1975, Condorcet, Raison et politique, Hermann, 1988.

BAUER M. et Bertin-Mourot B., 1996, Vers un modèle européen de dirigeants ? Abacus Editions, Biarritz.

BENTHAM J., 1791, Le panoptique, Belfond, 1977.

BOURDIEU P., 1978, postface à la traduction française d'E. Panofsky, Architecture gothique et pensée scolastique, Editions de Minuit.

CONDORCET, Esquisse d'un tableau historique des progrès de l'esprit humain, 1793, GF, 1988.

CROZIER M. et FrIEDBERG E., 1977, L'acteur et le système, Seuil, 1981.

DESROCHE H., 1993, « Humanismes et utopies », in Poirier (Jean) (sous la direction de), Histoire des Mours, La Pléiade (3 tomes), Tome 3.

DhOMBres J., 1987, in Fourcy A., 1828, Histoire de Polytechnique, Ambroise Belin, 1987.

DURKHEIM E., 1895-6, Le socialisme. Sa définition. La doctrine saint-simonienne, Retz-CEPL, 1978.

DURKHEIM E. et MAUSS M., 1903, «De quelques formes primitives de classification », in Mauss M., 1969, Oeuvres, Editions de Minuit, Tome 2.

DUVEAU G., 1961, Sociologie de l'utopie et autres essais, P.U.F.

ENGELS F. et MARX K., 1844-1880, Les utopistes, Maspero, 1976.

FISCHER R., 1993, «Eléments d'une histoire de la vision utopique du monde », Diogène, $\mathrm{n}^{\circ} 163$.

\footnotetext{
${ }^{1}$ DUVEAU, 1961
} 
FOURIER C., Vers la liberté en amour. Textes choisis et présentés par Daniel Guerin, Gallimard.

FRICK J.-P., 1988, «L'utopie de Saint-Simon : éléments d'une réflexion sur l'utopie saint-simonienne et sur la logique des utopies modernes », Revue Française de sciences politiques, 02-06.

KALAORA B. et SAVOYE A., 1989, Les inventeurs oubliés. Le Play et ses continuateurs, Champ Vallon, Seyssel.

KOYRE A., 1971, «Condorcet», in Etudes d'histoire de la pensée philosophique, Gallimard.

LACROIX J.-Y., 1994, L'utopie, Bordas.

MARCH J. G. et OLSEN J. P., 1991, « Le modèle du "garbage can" dans les anarchies organisées », in Décisions et organisations, Les éditions d'organisation.

MARTIN D., 1998, «La modernisation dans la crise : de Talcott Parsons à Jürgen Habermas », in Martin D. (éd.), Effets et méfaits de la modernisation des entreprises dans la crise, Desclée de Brouwer.

METZGER J.-L., 2000, Entre utopie et résignation: la réforme permanente d'un service public, L'Harmattan.

MiLls C. W., 1956, L'élite du pouvoir, Maspero, 1969.

MoRGAN N. S., 1996, “L'Utopie de Thomas More revisitée ”, Futuribles, janvier.

Musso P., 1997, Télécommunications et philosophies des réseaux, P.U.F..

Ozouf M, 1980, «L'utopie, encore et toujours », in Ozouf (M.), 1984, L'Ecole de la France. Essai sur la Révolution, l'utopie et l'enseignement, Gallimard.

PICON A., 1992, L'invention de l'ingénieur moderne, l'Ecole des Ponts et Chaussées 1747 - 1851, Presses de la fondation des Ponts et Chaussées.

Pouget M., 1998, Taylor et le taylorisme, P.U.F.

Ricoeur P., 1986, L'idéologie et l'utopie, Seuil, 1997.

RIHS C., Les philosophes utopistes, Marcel Rivière, 1970.

RUYER R., 1950, L'utopie et les utopies, Gérard Monfort, 1988.

SuleIMAN E. et MENDRAS H., 1995, Le recrutement des élites en Europe, La Découverte.

THOMAS J.-P., 1997, « Le résidu utopique », Raison présente, ${ }^{\circ} 121$.

TOURAINE A., 1965, Sociologie de l'action, Seuil.

VALADE B., 1992, «Culture », In Boudon (Raymond), Traité de sociologie, P.U.F. 